\title{
ENTEROCOCCAL BRAIN ABSCESS OF OTOGENIC ORIGIN: A CASE REPORT
}

Dr. K. Vidyasagar, Dr. R. Ravikumar. Dr. Nupur Pruthi.

1. Non-PG Senior Resident, Department of Neuromicrobiology, NIMHANS, Hosur Road, Bangalore.

2. Professor and Head, Department of Neuromicrobiology, NIMHANS, Hosur Road, Bangalore.

3. Assistant professor, Department of Neurosurgery, NIMHANS, Hosur Road, Bangalore

\section{CORRESPONDING AUTHOR}

Dr. R. Ravikumar MD,

Professor and Head, Department of Neuromicrobiology,

NIMHANS, Hosur Road, Bangalore-560029,

E-mail: ravikumarbly@yahoo.co.uk,

Ph: 09448073965.

ABSTRACT: Various aerobic and anaerobic bacteria have been reported as causative agents of brain abscess but only a few cases of enterococcal brain abscesses have been reported. We report a case of enterococcal brain abscess of otogenic origin in a 35 year old male who was known case of chronic suppurative otitis media (CSOM) and cholesteotoma of the right ear. The abscess material culture yielded an isolate which was identified as Enterococcus faecium by standard biochemical reaction. The isolate was sensitive to penicillin, ampicillin, gentamicin, vancomycin, ofloxacin, lincomycin, and cloxacillin.

KEY-WORDS: Brain abscess, Enterococcus faecium, Computerized tomography

INTRODUCTION: Despite the advent of modern neurosurgical techniques, new antibiotics and new powerful imaging technologies, brain abscess remains a potentially fatal central nervous system (CNS) infection.1-4 Brain abscess is a relatively uncommon infection with an incidence of $0.3-1.3 / 100,000$ persons per year 1 . Most of these cases occur in association with the predisposing conditions such as otitis media, sinusitis, or presence of pyogenic infections in other parts of the body. Dental infections are associated with around $2 \%$ of the brain abscesses. ${ }^{2}$

The causative pathogens of bacterial brain abscess vary with time period, geographic distribution, age, underlying medical and/or surgical conditions and mode of infection. A large number of Gram positive cocci, Gram negative aerobic bacilli, anaerobes and Mycobacterium tuberculosis have been reported as the causative agents of bacterial brain abscess. ${ }^{1-4}$ The commonest organisms causing brain abscess following an otogenic source include Staphylococcus aureus, Streptococcus pneumoniae, H. influenzae, Escherichia.coli, Proteus and Pseudomonas species. Very few studies have reported Enterococcus species as one of the causative agents of brain abscesses ${ }^{2-4}$.

We report a rare case of enterococcalbrain abscess of otogenic origin in a 35 years old male which was managed successfully with surgical drainage and antibiotics.

CASE HISTORY: A 35-year old male patient presented to the Department of Neurosurgery with three days of headache, giddiness and vomiting. The patient was a known case of CSOM and cholesteotoma of right ear for the past three years having ear discharge and ear pain. The patient underwent radical mastoidectomy with excision of cholesteotoma. On third post op day

Journal of Evolution of Medical and Dental Sciences/Volume1/ Issue3/July-Sept 2012 Page 254 
patient developed headache, vomiting and giddiness with blurring of vision with no h/o fever, seizures or limb weakness. At time of admission patient was conscious, had no fever and neck stiffness. Neurological examination revealed left hemi paresis with grade two power, bilateral papilledema, nystagmus and right cerebellar dysfunction. Cardiovascular, respiratory and abdominal examinations were found to be normal.

His white blood cell (WBC) count was $13000 / \mathrm{mm}^{3}$ with $70 \%$ neutrophils. The erythrocyte sedimentation rate (ESR) was $10 \mathrm{~mm} / \mathrm{hr}$. Biochemical parameters were within normal limits. CT of brain showed right cerebro-pontine angle cistern enhancing lesion with loculation of size $4.4 \times 3.5 \times 3.0 \mathrm{~cm}^{2}$ with mass effect on fourth ventricle. Intracranial mass or abscess was suspected as the probable diagnosis and craniotomy was performed and excision of right cerebro-pontine angle abscess with EVD placement was done. Abscess material was processed in the lab. Gram stain of the smear showed plenty of pus cells and Gram positive cocci in pairs and short chain along with Gram negativebacilli. ZN stain showed no acid fast bacilli. Abscess material was cultured on blood agar, MacConkey agar, thioglycollate broth and anaerobic blood agar aerobically and anaerobically. A culture was also put up in LJ medium. Culture yielded a bacterium which was identified as Enterococcus faecium by standard biochemical reactions ${ }^{6}$.

LJ medium showed no growth after eight weeks of incubation. The isolate was sensitive to penicillin, ampicillin, gentamicin, vancomycin, ofloxacin, lincomycin and cloxacillin by disc diffusion method. Patient was treated with iv ampicillin, iv gentamicin and metronidazole iv for 3 weeks. After three weeks of antibiotic therapy he was discharged without any neurological deficit. The patient on follow up visits was doing well with no residual neurological deficit and marked improvement in the radiological findings on a follow-up CT scan(fig1 B\&C).
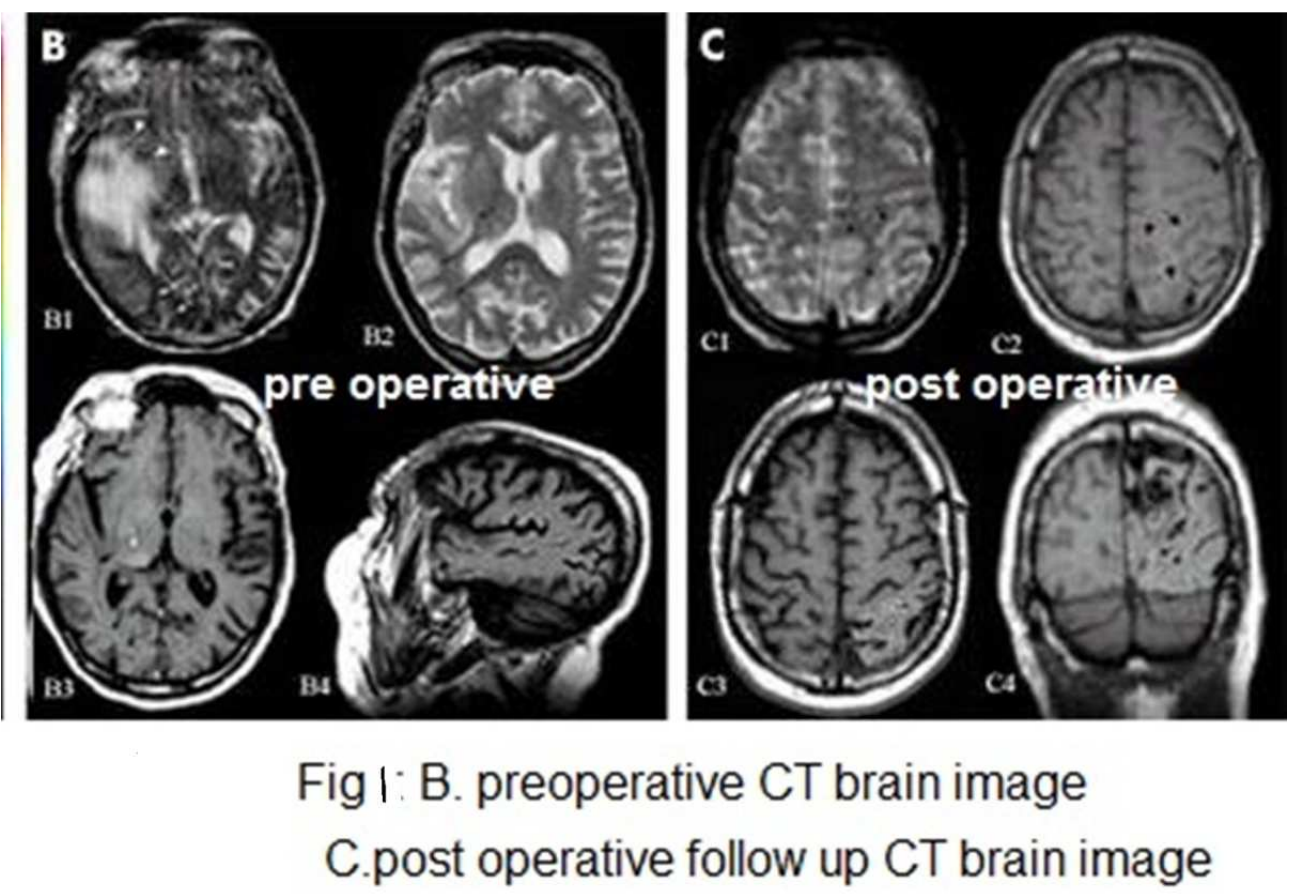
DISCUSSION: There have been many advances with respect to the diagnosis and management of brain abscess, resulting in a corresponding increase of survival rates. However its incidence is high, approximately $5 \%$ per million people, and the number of immuno-deficient hosts having high risk of opportunistic infections might be increasing. This disease continues to be one of the most common neurosurgical diseases ${ }^{[1-5]}$.

Regarding portal of entry, brain abscess is almost always secondary to a focus of suppuration elsewhere in the body or may develop either by a contiguous focus of infection, head trauma or hematogenous spread from a distant focus ${ }^{1-5}$ Thus the predisposing factors for the development of brain abscess include infections of the middle ear, mastoid, paranasal sinuses, orbit, face, scalp penetrating skull injury, intracranial surgery including insertion of ventriculo-peritoneal shunts ${ }^{5-6}$.

A previous study conducted by Malik et al.6from Mumbai showed that in 47 cases of brain abscess, the primary focus of infection could be established in 37 cases $(78.7 \%)$ and otogenic source was the commonest in $34 \%$ cases.

Another prospective study of pattern of brain abscess reported from India ${ }^{7}$ showed chronic suppurative otitis media to be the commonest predisposing factor in $48 \%$ of patients.

The location and number of abscesses depends upon the predisposing factors. The temporal abscess and the cerebellum are the commonest sites following otogenic source as observed in the present case. The list of bacteria causing brain abscesses is very large. It includes Gram negative aerobic bacilli like Klebsiella pneumoniae, Pseudomonas aeruginosa, Escherichia coli, Salmonella species, Proteus species, Klebsiella oxytoca, Haemophilus influenzae, Pasturella species, Vibrio cholerae non 01; Gram positive bacteria like Staphylococcus aureus, other Staphylococcus species, Streptococcus pneumoniae, Enterococcus species, Viridans streptococci, other Streptococcal species. The anaerobic organisms reported are Bacteroides species, Fusobacterium species, Peptostreptococcus and Propionibacterium species. ${ }^{1-4}$

The commonest organisms causing brain abscess following an otogenic source include Staphylococcus aureus, Streptococcus pneumoniae, H. influenzae, Escherichia.coli, Proteus and Pseudomonas species. ${ }^{1-7}$ Very few studies have reported Enterococcus species as one of the causative agents of brain abscesses in India.

Kurien et al.2in 1993 reported Enterococcus faecalis as one of the causative agents of brain abscesses in India, while studying 153 cases of brain abscesses.

In the year 2002, Park et al.4from Korea reported a case of otogenic brain abscess due to Enterococcusfaecium.

A recent case report from India by Mohanty et al. 3 in the year 2005 reported a brain abscess due to Enterococcus avium in a 19-year-old man with chronic otitis media since childhood.

Enterococcus faecalis and Enterococcus faecium are responsible for most enterococcal infections in humans, while Enterococcus gallinarum, Enterococcus avium and Enterococcus casseliflavus are not frequently reported ${ }^{8}$.Enterococciare clearly unusual etiological agents of brain abscess, and they account for $0.3-4 \%$ of reported cases of brain abscess ${ }^{9}$.Based on a clinical spectrum of symptoms and findings, enterococcal brain abscess can be found in two clinical forms postoperative and spontaneous. Postoperative brain abscess appears as a nosocomial infection usually associated with neurosurgical procedures and shunt devices ${ }^{10}$. Our patient had CSOM and also underwent radical mastoidectomy with excision of cholesteotoma which was considered as risk factors for his enterococcal brain abscess. For most clinical microbiological laboratories, the primary method of identifying Enterococcus species strains 
relies on phenotypic characterization [10]. The treatment of enterococcal brain abscess includes administration of appropriate antibiotics that have in vitro activity against the strain, and can efficiently penetrate the cerebrospinal fluid, in addition to the elimination of the predisposing factors if necessary ${ }^{10}$.

We would like to stress that a high index of suspicion, timely diagnostic support by CT scan, surgical intervention and vigorous antimicrobial therapy are crucial for better outcome.

\section{REFERENCES:}

1. Lu CH, Chang WN, Lin YC, Tsai NW, Liliang PC, Su TM, et al. Bacterial brain abscess: microbiological features, epidemiological trends, therapeutic outcomes. QJM. 2002; 95:501-09.

2. Kurien M, Job A, Mathew J, Chandy M. Otogenic intracranial abscess. Concurrent craniotomy and mastoidectomy- Changing trends in a developing country. Arch Otolaryngol Head Neck Surg. 1998; 124:1353-56.

3. Mohanty S, Dhawan B, Kapil A, Das BK, Pandey P, Gupta A. Brain abscess due to Enterococcus avium. Am J Med Sci. 2005; 329:161-62.

4. Park SY, Min JH, Ryu JW, Ko YS. Enterococcal otogenic brain abscess. Korean J Otolaryngol Head Neck Surg. 2002; 45:1188-92.

5. Sung DJ, Ealaan K, Chang YL, Kim IS, Son EIK, Kim DW, et al. Clinical features and surgical treatment of brain abscess. J Korean Neurosurg Soc. 2007; 41:391-96.

6. Malik S, Joshi SM, Kandoth PW, Vengsarkar US. Experience with brain abscesses. Indian Pediatr. 1994; 31:661-66.

7. Lakshmi V, Rao RR, Dinkar I. Bacteriology of brain abscess--observations on 50 cases. J Med Microbiol. 1993; 38:187-90.

8. Sood S, Malhotra M, Das BK, Kapil A. Enterococcal infections \& antimicrobial resistance. Indian J Med Res. 2008; 128: 111-21.

9. Kurup A, TeeW, Loo I, Lin R. Infection of central nervous system by motile Enterococcus: First case report. J Clin Microbiol.2001; 39:820-22.

10. Murray BE.The life and times of the Enterococcus. ClinMicrobiol Rev.1990;3: 46-65. 